\title{
Epilepsia y sueño
}

\section{Epilepsy and sleep}

\section{Viteri}

\section{RESUMEN}

La relación entre epilepsia y sueño se conoce desde hace más de un siglo. Al final del siglo XIX se observó una relación entre la aparición de las crisis epilépticas y el ciclo vigilia-sueño. Con la aparición de la electroencefalografía se comprobó cual era el efecto del sueño y de la privación del mismo sobre las descargas epileptiformes interictales y las crisis epilépticas. Existen una serie de síndromes epilépticos parciales y generalizados que se asocian con el sueño y que es importante reconocer para poder hacer un buen diagnóstico diferencial de los pacientes con trastornos motores y de comportamiento durante el sueño. En este artículo se revisan todos estos aspectos de la relación entre epilepsia y sueño.

Palabras clave. Epilepsia del sueño. Epilepsia del despertar. Ciclo vigilia-sueño.

An. Sist. Sanit. Navar. 2007; 30 (Supl. 1): 107-112.

\begin{abstract}
The relationship between sleep and epilepsy is known from more than a century ago. At the end of the XIX $^{\text {th }}$ century a temporal relationship between the onset of epileptic seizures and the wake-sleep cycle was observed. The introduction of the electroencephalogram allowed establishing the effect of sleep and sleeping deprivation on epileptiform interictal discharges and on epileptic seizures. The recognition of the variety of generalized and partial epileptic syndromes associated to sleep is important to make a correct differential diagnosis of patients presenting with motor and behavioural disturbances of sleep. This article reviews the different aspects of the relationship between epilepsy and sleep.
\end{abstract}

Key words. Sleep epilepsy. Wakefulness epilepsy. Wake-sleep cycle.
Departamento de Neurología y Neurocirugía. Clínica Universitaria de Navarra.

\section{Correspondencia:}

César Viteri Torres

Unidad de Epilepsias

Departamento de Neurología y Neurocirugía

Clínica Universitaria

Universidad de Navarra

Pamplona

E-Mail: cviteri@unav.es 
Las relaciones entre epilepsia y sueño son conocidas desde la antigüedad, ya están presentes en los escritos de Hipócrates y Aristóteles. El interés moderno por las epilepsias nocturnas se desarrolla desde finales en el siglo XIX, pudiéndose delimitar dos etapas: la clínica, en la que hasta hace pocas décadas los conocimientos se basaban solo en las observación clínica; y más recientemente, la etapa de desarrollo de la electroencefalografía y la disponbilidad de monitorización prolongada y de los estudios polisomnográficos ${ }^{1,2}$.

\section{Relación entre la aparición de crisis y el ciclo vigilia-sueño}

Antes del electroencefalograma (EEG), la atención de los investigadores se concentraba en la relación entre las crisis, la hora del día y el sueño. La mayoría de observaciones se realizaron en pacientes ingresados en instituciones con horarios rígidos. Féré estudió la hora de aparición de las crisis a lo largo de 3 meses en pacientes epilépticos hospitalizados, encontrando que 1.285 de 1.985 crisis ocurrieron entre las 8 de la tarde y las 8 de la mañana. Gowers (1885) en 850 pacientes institucionalizados observó que el $21 \%$ de crisis ocurrían exclusivamente durante la noche (epilepsias nocturnas), $42 \%$ crisis solo durante el día (epilepsias diurnas) y $37 \%$ crisis indistintamente de día o de noche. LangdonDown y Brain (1929) analizaron durante 6 meses la hora de presentación en 2.524 convulsiones de 66 pacientes institucionalizados: el $24 \%$ de las crisis eran nocturnas, $43 \%$ diurnas y $33 \%$ ocurrían al azar.

Janz describió por primera vez la que llamó "epilepsia del despertar" este autor revisó cinco artículos sobre la cronobiología de las crisis generalizadas tónico-clónicas (CGTC) ${ }^{4}$. Entre 2.825 pacientes, $44 \%$ tenían las crisis durante el sueño, en ellas el origen era con más frecuencia temporal y $60 \%$ presentaban lesiones estructurales; 33\% de las crisis ocurrían al despertar, de ellas $12,5 \%$ tenían carácter familiar; y el 23\% restante al azar. Gibberd y Bateson ${ }^{5}$ estudiaron la historia natural de las epilepsias relacionadas con el sueño en $12 \%$ de 645 pacientes, encontraron que el $30 \%$ de epilepsias del sueño se vuelven difusas o del despertar mientras que otro $30 \%$ remiten. Los estudios clínicos confirmaron que las crisis relacionadas con el sueño tienen un patrón ultradiano diferenciado al inicio y final del sueño, mientras que las crisis en la epilepsia diurna ocurren preferentemente al despertar o a media tarde.

\section{Efecto del sueño sobre las descargas epileptiformes interictales (DEI)}

Gibbs y Gibbs $^{6}$ observaron en 500 pacientes que mientras el $36 \%$ presentaban descargas epileptiformes interictales en vigilia, $82 \%$ lo hacían durante el sueño, comprobando que el sueño activa focos independientes en algunos pacientes, y subrayaron que la probabilidad de activar DEI era máxima en pacientes con crisis psicomotoras y menor en aquellos con petit mal.

El sueño NREM activa las DEI en las epilepsias parciales, así lo comprobaron Gloor et $\mathrm{al}^{7}$ (1958) en 300 pacientes, de los cuales 57\% tenían más DEI durante el sueño. Niedermeyer y Rocca ${ }^{8}$ por su parte vieron que alrededor del $30 \%$ de pacientes con epilepsia del lóbulo temporal refractaria tenían DEI sólo durante el sueño. El EEG se caracteriza por un aumento de la frecuencia de puntas al comienzo del sueño, que alcanza el máximo en el sueño lento y decae en la fase REM. Al mismo tiempo el campo del foco interictal aumenta.

En las epilepsias idiopáticas generalizadas el sueño aumenta las DEI presentes en vigilia tanto en crisis de ausencia como en CGTC. El aumento de DEI en pacientes con CGTC ocurre en la fase de sueño NREM. La frecuencia de puntas aumenta al entrar en sueño, crece durante el sueño lento y decae en REM. La frecuencia aumenta bruscamente después de despertar. El efecto del sueño sobre la punta-onda a $3 \mathrm{~Hz}$ es más conflictivo, durante el sueño NREM se vuelve más irregular, mezclada con polipuntas y ocurre en trenes semiperiódicos, en cambio durante el sueño REM se parece a la vigilia.

\section{Bases fisiopatológicas}

La activación de las DEI durante el sueño NREM representa un estado de sincronización del EEG con relativa preserva- 
ción del tono muscular antigravitatorio. Las oscilaciones sincrónicas de las neuronas corticales que generan los husos de sueño, los complejos $\mathrm{K}$ y las ondas lentas tónicas de fondo durante el sueño NREM, promueven la propagación de las crisis durante esta parte del sueño. En contraste, el sueño REM se caracteriza por la desincronización del EEG y pérdida del tono muscular. La desincronización del EEG impide la propagación de las crisis durante el sueño REM, lo mismo que en la vigilia, y la ausencia de tono muscular bloquea la expresión clínica de las crisis ${ }^{2}$.

\section{Efecto de la privación de sueño sobre las crisis epilépticas y las DEI}

La privación total de sueño (más de 24 horas seguidas sin dormir) es reconocida como factor activador de las crisis por el 18 al $24 \%$ de pacientes adultos con epilepsia. También la privación parcial de sueño actúa como activador de las crisis. Así en un estudio sobre el efecto de la reducción del periodo normal de sueño al menos 1,5 horas de la media de sueño, demostró que una pérdida moderada de sueño puede precipitar crisis ${ }^{9}$. No está claro si la activación de las DEI ocurre por un efecto activador directo del sueño o por un efecto activador independiente de la privación de sueño.

\section{Efecto de la epilepsia sobre el sueño}

Las crisis epilépticas rompen la regulación normal del ciclo vigilia-sueño. Así, las crisis nocturnas frecuentes producen alteraciones significativas del sueño, los pacientes tienen más inestabilidad del sueño y más despertares espontáneos antes del inicio del tratamiento antiepiléptico. La traducción clínica de las crisis durante el sueño puede ser una sensación de falta de descanso significativa. En cuanto al efecto de las crisis sobre la arquitectura del sueño, los efectos más prominentes son la somnolencia postictal y el insomnio. Las crisis durante el sueño y el estado postictal producen fragmentación del sueño y supresión del sueño REM, estas alteraciones postcríticas pueden extenderse a las noches siguientes.

\section{Epilepsias del sueño o del despertar}

En la actualidad se admite que varios síndromes epilépticos se caracterizan por crisis que ocurren de forma predominante o exclusiva durante el sueño o al desper$\operatorname{tar}^{2}$ (Tabla 1).

\section{Epilepsia con crisis generalizadas tónico-clónicas del despertar}

La mayoría de CGTC de las epilepsias generalizadas idiopáticas se presentan después de despertar, y particularmente cuando la persona es despertada tras un periodo de privación de sueño seguido de un sueño breve. En la clasificación revisada de las epilepsias y síndromes epilépticos de 1989 consta un síndrome específico con CGTC relacionadas con el sueño: el síndro-

Tabla 1. Epilepsias del sueño y del despertar ${ }^{2}$

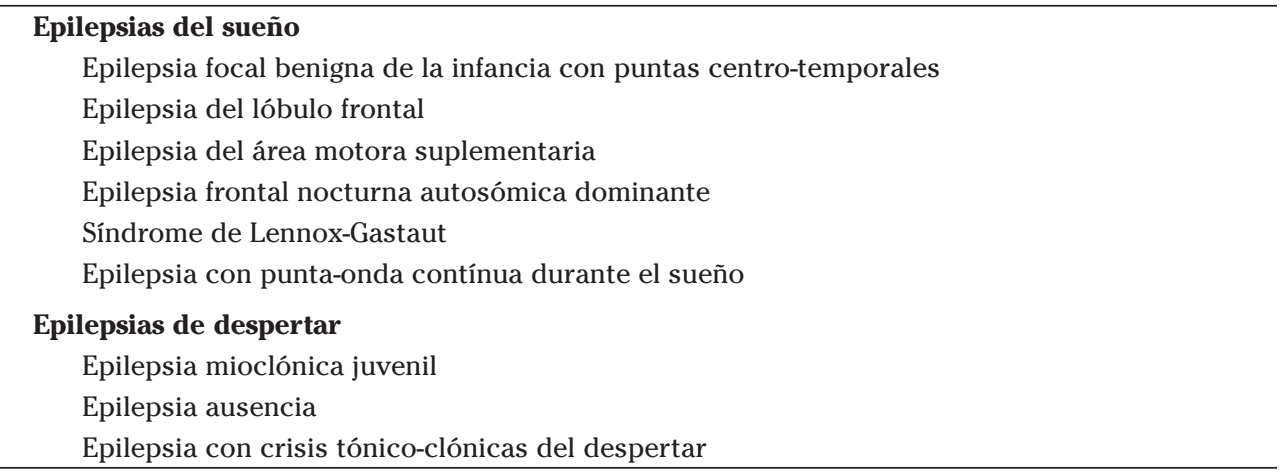


me de Epilepsia de Gran Mal del Despertar ${ }^{10}$. Los pacientes cuya única manifestación de su epilepsia son CGTC que no guardan relación con el despertar se clasifican como epilepsia generalizada idiopática con crisis generalizadas tónico-clónicas.

Janz y Wolf han revisado a fondo este síndrome $^{11,12}$. Consideran a Gowers (1885) como el primer autor que menciona a pacientes cuyas crisis ocurren sólo por la mañana temprano, que constituyen el 5\% de sus casos, y señalan que otros autores como Langdon-Down y Brain (1929), Patry (1931), Hopkins (1933) o Griffiths y Fox (1938) confirmaron esta observación.

$\mathrm{Janz}^{3}$ en 1953 publicó el primer artículo sobre la que denominó "epilepsia del despertar", consistente en una epilepsia idiopática con CGTC, y señaló que algunos pacientes tenían un segundo pico de crisis, más a menudo al final de la tarde o la noche, en momentos de relajación. Loise$\mathrm{au}^{13}$ revisó la experiencia francesa y corroboró la existencia de un síndrome de epilepsia del despertar con crisis generalizadas tónico-clónicas, a veces asociado a crisis mioclónicas y ausencias, con descargas generalizadas epilépticas en el EEG.

Según la Clasificación de Epilepsias y Síndromes Epilépticos de la Liga Internacional contra la Epilepsia ${ }^{10}$, la epilepsia con crisis generalizadas tónico-clónicas al despertar es un síndrome que se inicia más frecuentemente en la segunda década de la vida. Las CGTC ocurren exclusiva o predominantemente (más del $90 \%$ de las veces) poco después de despertar, con independencia de la hora del día, o en un segundo pico, en el periodo de relajación vespertina. Cuando ocurren otras crisis son principalmente ausencias o crisis mioclónicas, como en la epilepsia mioclónica juvenil. Las crisis pueden ser precipitadas por privación de sueño u otros factores externos. La predisposición genética es relativamente frecuente. El EEG muestra uno de los patrones de epilepsia generalizada idiopática y existe una correlación significativa con fotosensibilidad.

De acuerdo con Janz ${ }^{12}$, para diagnosticar con certeza este síndrome hace falta que ocurran como mínimo 6 CGTC, exclusiva o mayoritariamente durante el sueño, a cualquier hora del día, o durante momentos de ocio o relajación. La definición del término predominante como "más del $90 \%$ del tiempo" que propone la clasificación de la liga Internacional contra la Epilepsia ${ }^{10}$ ha sido ampliada por otros investigadores con un criterio más flexible, a "más de la mitad del tiempo"12.

Los estudios revisados por Wolf ${ }^{11}$ que recogen los datos de distribución por sexos muestran una ligera preponderancia masculina. Una historia familiar positiva está presente en $90(12,5 \%)$ de los 719 pacientes estudiados por Janz ; 73 de 946 pacientes tenían historia familiar positiva cuando las CGTC ocurrían durante el sueño $(7,7 \%)$ y solo 17 de $445(3,8 \%)$ cuando la aparición de las crisis era aleatoria. En la serie de Tsuboi y Christian ${ }^{14} 16$ de 147 pacientes con CGTC al despertar (10,9\%) tenían predisposición familiar en contraste con el $6,7 \%$ de pacientes con CGTC durante el sueño. En estudios más recientes, los hallazgos no fueron concluyentes ${ }^{15}$, o se ha observado una relación con el locus EJM-1, la misma que no está presente en la epilepsia del adolescente con CGTC en cualquier momento de la vigilia ${ }^{16}$.

La edad de comienzo tiene un amplio rango, mayor que en otros síndromes epilépticos generalizados idiopáticos. Existe un claro pico de la edad de comienzo hacia la pubertad, entre los 16 y 17 años. Más del $80 \%$ de pacientes tienen su primera crisis en la segunda década de la vida ${ }^{11,17}$.

Cuadro clínico. Está constituído por crisis generalizadas tónico-clónicas. Las CGTC puras, sin ausencias o mioclonías previas, son infrecuentes. Las crisis de ausencia o mioclónicas pueden ser leves y a veces pasan desapercibidas para el paciente y son imperceptibles para el observador. Para detectarlas es preciso realizar una historia clínica muy meticulosa o disponer de un registro vídeo-EEG ${ }^{17}$.

La relación entre las crisis y el ritmo vigilia-sueño que define a este síndrome debe establecerse con la primera manifestación clínica, sea ausencia, mioclonía o crisis convulsiva, y considerar el conjunto de crisis como un evento único. Cuando las crisis no convulsivas son prolongadas, 
la relación de la CGTC con el despertar puede quedar oscurecida y aparentar que no guardan relación ${ }^{11}$.

Exploración física. No se han descrito hallazgos patológicos físicos o mentales en los pacientes con CGTC al despertar, si bien Janz ${ }^{11}$ describe a estos pacientes desde el punto de vista psicológico como: "inestables, no fiables, desconsideradamente negligentes en sus obligaciones y en sus propios intereses, siempre dispuestos a caer en la más ligera tentación aún en contra de su mejor juicio", y señala que muchos pacientes son indisciplinados, que cumplen mal el tratamiento, con una conducta de sueño irregular que puede favorecer la aparición de crisis.

Factores desencadenantes. Las crisis pueden ser provocadas por privación de sueño, consumo de alcohol o fatiga excesiva. Algunos pacientes identifican factores estresantes, pero el análisis detallado suele demostrar que dichos factores actúan a través de una alteración del sueño. Conforme aumenta la edad, las crisis tienden a ser más frecuentes y los factores exógenos desencadenantes menos importantes. En contraste con otras EGI, la epilepsia con CGTC al despertar está relacionada con la fotosensibilidad ${ }^{11}$.

Electroencefalograma. En un 50-70\% de los casos se observa punta-onda o polipunta-onda en forma de descargas generalizadas. Rara vez se encuentran alteraciones focales sin descargas generalizadas. El $13 \%$ de los casos muestran fotosensibilidad en el EEG ${ }^{18,19}$. Cuando el EEG de rutina es normal, se debería realizar un registro vídeo-EEG del sueño y del despertar.

Diagnóstico diferencial. Se realiza con pacientes que presentan otras epilepsias generalizadas idiopáticas con CGTC al despertar ${ }^{17}$. Hay un claro solapamiento entre los síndromes de epilepsia mioclónica juvenil, epilepsia ausencia juvenil, epilepsia de Grand Mal al despertar y epilepsia con ausencias de la infancia. Cada una de estas entidades puede cursar con un único tipo de crisis o combinar distintos tipos de crisis.

Diagnóstico. Por definición, el síndrome requiere que la gran mayoría de las crisis ocurran dentro de las 2 horas siguien- tes al despertar (a cualquier hora del día); un segundo pico de frecuencia puede aparecer durante la relajación del final del día. Como ya se ha señalado, Janz ${ }^{12}$ establece que el diagnóstico definitivo exige al menos seis crisis predominantemente en el despertar o en momentos de relajación. Andermann y Berkovic ${ }^{20}$ señalan que esta condición es difícil de cumplir en la actualidad y que ante un paciente con este número de crisis, teniendo en cuenta la disponibilidad de varios tratamientos eficaces, habría que pensar que tiene una forma grave de epilepsia, que no cumple adecuadamente el tratamiento o las dos cosas. La dificultad para identificar el síndrome en su inicio probablemente explica por qué en el General Practice Study of Epilepsy realizado en el Reino Unido ${ }^{21}$, en 594 nuevos diagnósticos no hubo ningún caso de epilepsia con CGTC al despertar.

Tratamiento y pronóstico. El tratamiento de primera línea debe ser monoterapia con valproato sódico o lamotrigina ${ }^{17}$. Otros fármacos eficaces son: levetiracetam, topiramato y zonisamida. Entre los fármacos clásicos son eficaces fenobarbital y primidona. En la mayoría de los casos el control de las crisis es total, si bien la epilepsia con crisis de gran mal al despertar mantiene a lo largo de la vida una alta incidencia de recurrencia $(83 \%)$ en caso de retirada del tratamiento, significativamente mayor que en otras epilepsias con CGTC.

Algunos fármacos antiepilépticos como carbamacepina u oxcarbazepina están relacionados con el empeoramiento de epilepsias generalizadas idiopáticas juveni$\mathrm{les}^{22}$, incluyendo aumento de frecuencia de las crisis existentes y la aparición de nuevos tipos de crisis. Este efecto paradójico parece debido a características farmacodinámicas y no se debe confundir con fluctuaciones espontáneas de las crisis o mal cumplimiento del tratamiento o un efecto.

En el momento actual, la identidad del síndrome de crisis de gran mal al despertar es controvertida y probablemente desaparecerá como entidad individualizada. El principal motivo es que las CGTC relacionadas con el despertar pueden aparecer en varias epilepsias generalizadas 
idiopáticas, con manifestaciones clínicas, respuesta al tratamiento y pronóstico variables. Actualmente es más difícil identificar la relación de las crisis con el sueño porque el tratamiento precoz en la mayoría de pacientes las elimina cuando solo han padecido unas pocas crisis, sin dar tiempo a establecer la cronobiología.

\section{BIBLIOGRAFÍA}

1. PASSOUANT P. Historical aspects of sleep and epilepsy. Epilepsy Res Suppl 1991; 2: 19-22.

2. FoldVARY-Schaefer N, GRIGG-DAMBERGer M. Sleep and epilepsy: what we know, don't know, and need to know. J Clin Neurophysiol 2006; 23: 4-20.

3. JANZ D. "Aufwach"-Epilepsien (als Ausdruck einer den "Nacht-oder Schlaft"-Epilepsien gegenüberstehenden Werlaufsform epileptischer Erkrankungen). Arch Psychatr Nervenkr 1953; 191: 73-98.

4. Janz D. Die epilepsien. Spezielle pathologie und therapie. Stuttgart: Thieme; 1969.

5. GibBerd FB, Bateson MC. Sleep epilepsy: its pattern and prognosis. BMJ 1974; 25: 403405.

6. GIBBS EL, GIBBS FA. Diagnostic and localizing value of electroencephalographic studies in sleep. Nerv Ment Dis 1947; 26: 336-376.

7. Gloor P, Tsai C, HAdDAD F. An assessment of the value of sleep-electroencephalography for the diagnosis of temporal lobe epilepsy. Electroencephalogr Clin Neurophysiol 1958; 10: 633-649.

8. Niedermeyer E, Rocca U. The diagnostic significance of sleep electroencephalograms in temporal lobe epilepsy. Eur Neurol 1972; 7: 119-129.

9. RAJNe P, VERES J. Correlations between night sleep duration and seizure frequency in temporal lobe epilepsy. Epilepsia 1993; 34: 579.

10. Commission on classification and terminology of the International League Against E. Proposal for revised classification of epilepsies and epileptic syndromes. Epilepsia. 1989; 30: 389-399.

11. Wolf P. Epilepsy with grand mal on awakening. In: Roger J, Bureau M, Dravet C, Genton P, Tassinari CA, Wolf P, editors. Epileptic syndromes in infancy, childhood and adolescence. 3rd ed. Eastleigh: John Libbey \& Co Ltd; 2002; 357-367.

12. JANZ D. Epilepsy with grand mal on awakening and sleep-waking cycle. Clin Neurophysiol 2000; 111: 102-110.

13. LOISEAU P. Crises épileptiques survenant au réviel et épilepsie du réveil. Sud Méd Chirugical 1964; 99: 11492-11502.

14. Tsuboi T, Christian W. Epilepsy: a clinical, electroencephalographic and statistical study of 466 patients. Berlin. Springer 1976.

15. Delgado-Escueta AV, GreEnBerg D, Weissbecker $\mathrm{K}$, LiU A, Treiman L, Sparkes $\mathrm{R}$ et al. Gene mapping in the idiopathic generalized epilepsies: juvenile myoclonic epilepsy, childhood absence epilepsy, epilepsy with grand mal seizures, and early childhood MYOCLONIC epilepsy. Epilepsia 1990; 31: 19.

16. GreEnBerg DA, Durner M, Resor S, Rosenbaum D, SHINNAR S. The genetics of idiopathic generalized epilepsies of adolelescent onset: differences between juvenile myoclonic epilepsy and epilepsy with random grand mal and with awakening grand mal. Neurology 1995; 45: 942.

17. Panayiotopoulos CP. Idiopathic generalised epilepsy with generalised tonic-clonic seizures only. A clinical guide to epileptic syndromes and their treatment. Chipping Norton: Bladon Medical Publishing 2002; 145-147.

18. Betting LE, Mory SB, Lopes-Cendes I, Li LM, GuERreiro MM, GuERrEIRo CA et al. EEG features in idiopathic generalized epilepsy: clues to diagnosis. Epilepsia 2006 Mar; 47: 523-528.

19. Fittipaldi F, Curra A, Fusco L, Ruggieri S, MANFREDI M. EEG discharges on awakening: a marker of idiopathic generalized epilepsy. Neurology 2001; 56: 123-126.

20. Andermann F, Berkovic SF. Idiopathic generalized epilepsy with generalized and other seizures in adolescence. Epilepsia 2001; 42: 320.

21. HART YM, SANDER JWAS, Johnson AL, SHorvon SD. National General Practice Study of Epilepsy: recurrence after a first seizure. Lancet 1990; 336: 127-1274.

22. Gelisse P, Genton P, Kuate C, Pesenti A, BaldyMolulinier M, CRespel A. Worsening of seizures by oxcarbazepine in juvenile idiopathic generalized epilepsias. Epilepsia 2004; 45: 1282-1286. 